\title{
La migración de personas de las comunidades a la ciudad
}

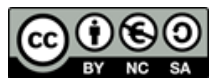

\section{The migration of people from communities to the city}

\author{
Efraín Velasteguí López. ${ }^{1}$
}

Recibido: 08-03-2018 / Revisado: 15-03-2018 / Aceptado: 20-03-2018 / Publicado: 05-04-2018

\section{Resumen. $\quad$ DOI: https://doi.org/10.33262/concienciadigital.v1i2.948}

La migración es un problema social en cualquier parte del mundo en el ecuador en los últimos años la migración ha aumentado, siendo ocasionados por falta de condiciones económicas la falta de oportunidad de trabajo que han llevado a la pobreza y haciendo que los padres de las familias salgan hacia las ciudades u otros países con la esperanza de buscar una mejor calidad de vida y así poder brindad una día mejor a su familia, pero ellos no se han dado cuenta de los serios problemas que desencadena la migración tales como la desintegración familiar, maltrato, abandono, trastornos emocionales, alcoholismo, problemas sociales tales como delincuencia, drogadicción, parte de las personas que quedan encargados del bienestar de sus hijos estos problemas aumentan cada día debido a la migración esto se debe también que en las comunidades rurales no hay fuentes de empleo por esta razón ellos optan por viajar a las grandes ciudades dos de las más grande ciudades del Ecuador donde se han visto más personas migrantes son en Guayaquil y Quito son ciudades donde la mayoría de personas de las comunidades rurales se han concentrado buscando nuevas oportunidades de empleo así poder dar un nivel de vida mejor de la que ellos tenían en las comunidades pero muchas de las veces esa emigración ha traído consecuencias como la forma de vida en las ciudades son peor porque no cuentas de buenos servicios básico o simplemente son discriminados por los grandes terratenientes que explotan en este caso a los indígenas de la sierra ecuatoriana.

Palabras claves: Migración, Emigración, Inmigración, Huasipungo

\footnotetext{
${ }^{1}$ Ciencia digital, Ambato, Ecuador, luisefrainvelastegui@cienciadigital.org
} 


\section{Abstract.}

Migration is a social problem in any part of the world in the Ecuador in recent years migration has increased, being caused by lack of economic conditions the lack of work opportunities that have led to poverty and causing the parents of families go to the cities or other countries with the hope of seeking a better quality of life and thus be able to provide a better day for their family, but they have not realized the serious problems that the migration triggers such as the family disintegration, mistreatment, abandonment, emotional disorders, alcoholism, social problems such as delinquency, drug addiction, part of the people who are responsible for the welfare of their children these problems increase every day due to migration this is also due to the fact that in rural communities there are no sources of employment for this reason they choose to travel to the big cities two of the largest cities in Ecuador where have seen more migrant people are in Guayaquil and Quito are cities where most people from rural communities have concentrated looking for new employment opportunities to be able to give a better standard of living than they had in the communities but many times that emigration has brought consequences as the way of life in the cities are worse because they do not count on good basic services or they are simply discriminated against by the big landowners who exploit in this case the indigenous people of the Ecuadorian highlands

Keywords: Migration, Emigration, Immigration, Huasipungo.

\section{Introducción.}

Según los autores (Cerrutti 2005, Hidalgo 2004, Altamirano 2003) Las migraciones de personas son el desplazamiento, el migrante se afinca permanentemente en el lugar de inmigración. Si hay retorno éste es solamente temporal lo cual quiere decir que la ruptura con el lugar de origen es bastante radical. Los vínculos afectivos económicos o sociales que siempre perduran entre el migrante y su antiguo entorno social, hacen del migrante un habitante diferente al nativo. El afincamiento frecuentemente es resultado de una consolidación de anteriores movimientos temporales, rara vez es únicamente individual sino más bien familiar. Al adjetivo "definitivas" no debe dárselo su significado estricto; no quiere decir que después de la migración no habrá otra en el futuro. El fenómeno de la migración tiene un carácter mundial e histórico. Desde que los grupos humanos surgen, o más bien desde que el hombre aparece en la tierra, inicia su peregrinar para tratar de obtener alimentos, así como refugio y protección de las inclemencias de la naturaleza. Las migraciones son desplazamientos de grupos humanos que los alejan de sus residencias habituales. Esta definición engloba fenómenos demográficos muy diversos, puede referirse a movimientos pacíficos o agresivos, voluntarios o involuntarios. Primer Congreso Internacional de Educación "Construyendo inéditos viables" Área Temática Educación y multiculturalismo 1817 La humanidad que en el pasado se desparramó por el planeta ocupando paulatinamente ámbitos de riquezas promisorios y tenue demografía, está regresando atropelladamente a 
formar grandes grupos urbanos las migraciones del campo a la ciudad, se están produciendo en todo el mundo a un ritmo extraordinario.

Desplazamientos de personas que toman la determinación de trasladarse a otro lugar sin presiones externas y con muy diversos fines. Por falta de presión externa nos referimos a que no está en peligro su supervivencia o la de su familia, no existe una amenaza contra su vida o la de sus dependientes. Bajo este rubro encontramos diversos tipos de migración, como las que ocurren por razones económicas, demográficas y en especial, por razones sociales y culturales. Entre estas se encuentra la búsqueda de mejor educación.

Según mi criterio la migración de personas del campo a la ciudad se debe a diversos motivos que serían para mejor la calidad de vida una buena educación a sus hijos la mayoría de personas del sector rural han optado por viajar a las grande ciudades del país se dice que las dos ciudades más grandes donde se concentran la mayor parte de emigrantes son Guayas y Pichincha son dos ciudades más grandes del ecuador donde también se concentra la mayor cantidad de migrantes aunque una buena parte de ecuatorianos han salido del países a diferentes países de Europa para buscar nuevas fuentes de empleo se dice que a partir de la segunda mitad de la década de 1950, Estados Unidos aparece como uno de los nuevos y mejores destinos para la migración principalmente eso se debía que la influencia de la revolución industrial surge como blanco para la migración de los primeros profesionales ecuatorianos, además algunos trabajadores manuales especializados en industria textil en construcciones civiles otros trabajadores viajaron a empresas como United Fruit era una transnacional que operaba en los campos bananeros aquí en el ecuador ellos exportaban a diferentes países de Europa muchas de las personas viajaban como operadores y se quedaban en esos países, esto explica porque los primeros ecuatorianos que migraron se han radicado en Europa en estos últimos años habido más emigración de personas ya sea a las ciudades o al extranjero en esta Figura N.-1 podremos observar el número de salidas y entrada de ecuatorianos que viajan hacia el extranjero o regresan otra vez a nuestro país.

Figura N 1 Número de entradas y salidas de ecuatorianos Periodo 1997 - 2017

En el año 2017 los ecuatorianos registraron 1.506 .290 entradas y 1.547 .312 salidas

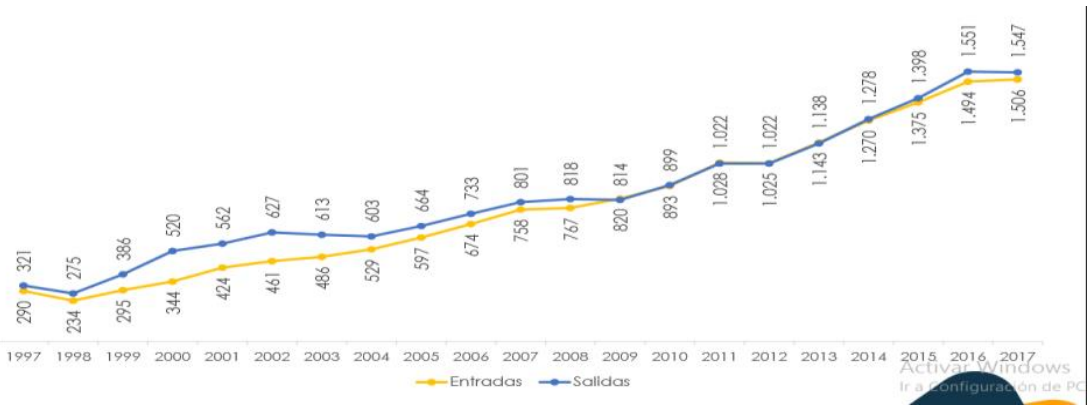

Fuente: Registro Estadístico de Entradas y Salidas Internacionales 1997- 2017 
Según los autores (Instituto Nacional de Estadística y Censos 2018, Facso Andes 2008, Naciones Unidas 2013) los últimos datos publicados Ecuador tiene 1.131.427 emigrantes, lo que supone un $6,81 \%$ de la población de Ecuador la migración internacional se ha convertido en uno de los hechos más importantes para el Ecuador en el cambio de siglo. El éxodo de más de un millón de ecuatorianos en los últimos diez años ha significado cambios sociales, económicos y culturales; cuyos impactos a nivel local, nacional y regional no han sido todavía lo suficientemente evaluados en el país en numerosas ocasiones los Estados miembros han pedido que se mejore la evidencia empírica sobre la migración internacional y sus vínculos con el desarrollo, no obstante, desde 2010 el crecimiento de la población migrante se ha ralentizado. Tras la crisis económica mundial, el crecimiento anual de la población migrante mundial disminuyó aproximadamente 3,6 millones en la siguiente tabla podemos observar tasa de migrantes, emigrantes ya sea hombres o mujeres se puede mostrar también los años.

Tabla N 1 Migración en el Ecuador

\begin{tabular}{|l|c|c|c|c|}
\hline Año & Emigrantes hombres & Emigrantes mujeres & Emigrantes & \% Emigrantes \\
\hline 2017 & 535.545 & 595.882 & 1.131 .427 & $6,81 \%$ \\
\hline 2015 & 524.323 & 580.857 & 1.105 .180 & $6,79 \%$ \\
\hline 2010 & 556.913 & 596.986 & 1.153 .899 & $7,69 \%$ \\
\hline 2005 & 479.550 & 508.467 & 988.017 & $7,20 \%$ \\
\hline 2000 & 217.484 & 228.280 & 445.764 & $3,56 \%$ \\
\hline 1995 & 159.426 & 166.160 & 325.586 & $2,81 \%$ \\
\hline 1990 & 105.553 & 108.178 & 213.731 & $2,05 \%$ \\
\hline
\end{tabular}

Fuente: Instituto de Estadísticas y Censo

Según mi criterio la migración en el ecuador ha aumentado siendo la mayor parte de ecuatorianos en el extranjero ya sea hombres mujeres según los datos estadístico de INEC cada año la migración aumenta ya sea por diferentes causas que pueden ser la falta de empleo la principal causa de la salida de personas hacia el extranjero es para poder buscar fuentes de empleo para tener un nivel de vida mucho mejor de la que tiene en el área rural mediante el cuadro podemos observar que la migración de personas desde 1990 ha ido incrementando anualmente esto quiere decir que en nuestro las fuentes de empleo son escasas o la forma de vida de la población no son acorde a sus necesidad por ese motivo ellos optan por salir del 
país para poder buscar nuevos rumbos para salir ya sea de la pobreza así para poder tener una mejor educación para sus hijos o dar un nivel de vida estable para su familia.

Tabla N 2. Población de migrantes internacionales (en millones)

\begin{tabular}{lrrrr} 
& $\mathbf{1 9 9 0}$ & $\mathbf{2 0 0 0}$ & $\mathbf{2 0 1 0}$ & $\mathbf{2 0 1 3}$ \\
\hline Mundo & $\mathbf{1 5 4 , 2}$ & 174,5 & 220,7 & $\mathbf{2 3 1 , 5}$ \\
Regiones desarrolladas & 82,3 & 103,4 & 129,7 & 135,6 \\
Regiones en desarrollo & 71,9 & 71,1 & 91 & 95,9 \\
África & 15,6 & 15,6 & 17,1 & 18,6 \\
Asia & 49,9 & 50,4 & 67,8 & 70,8 \\
Europa & 49 & 56,2 & 69,2 & 72,4 \\
América Latina y el Caribe & 7,1 & 6,5 & 8,1 & 8,5 \\
América del Norte & 27,8 & 40,4 & 51,2 & 53,1 \\
Oceanía & 4,7 & 5,4 & 7,3 & 7,9 \\
\hline
\end{tabular}

Fuente: Naciones Unidas (2013), Trends in International Migrant Stock: The 2013 Revision.

En este cuadro podemos observar los datos estadísticos de la migración desde el año de 1990 hasta el 2013 se puede ver de los diferentes continentes con cifras numéricas.

\section{Las comunidades}

Según los autores (Causse Cathcart 2009, Marchioni 1937, Valle 2002) las comunidades son conjuntos de hablantes que comparten efectivamente, al menos, una lengua, pero que además comparten un conjunto de normas y valores de naturaleza sociolingüística: comparten unas mismas actitudes lingüísticas, unas mismas reglas de uso, un mismo criterio a la hora de valorar socialmente los hechos lingüísticos, unos mismos patrones sociolingüísticos, la comunidad y su grado de heterogeneidad para que sea realmente una comunidad de habla. De esta forma, resume que una comunidad de habla "es básicamente una comunidad de consenso, de sintonía entre grupos e individuos diferentes, donde el conflicto está minimizado no hay dos comunidades iguales así como no hay dos personas iguales, cada comunidad tendrá que realizar su propio e inimitable proceso particular. Por ello no sirven en esta tarea "modelos" rígidos y únicos, sino experiencias de otras situaciones que pueden ser útiles, se entiende por comunidad "un territorio concreto, con una población determinada, que dispone de determinados recursos y que tienen determinadas demandas", una comunidad siempre es el conjunto de estos cuatro factores territorio, población, recursos y demandas y sus mutuas, constantes y mutantes interrelaciones. Aislar el proceso en uno sólo de estos factores lleva al fracaso, el proceso comunitario sea de organización, sea de desarrollo, sea de los dos juntos e integrados no es algo espontáneo, alguien tiene que tomar la iniciativa y siempre es trabajo profesional, aunque cuente, como es natural, con el voluntariado.

Según mi criterio diría que las comunidad son poblaciones pequeñas donde la gente trabajan con un solo fin para poder hacer que la población día a día prospere ya sea económica o socialmente para tener un pueblo unido las comunidad también se podría decir que son un 
conjunto de habitantes que tienen los mismos idiomas, costumbres, tradiciones, fiestas, formas de vestir, de trabajar el terreno y producir alimentos, las comunidades en mayor parte están alejadas de la urbe y están ubicados en zonas rurales donde ellos viven en paz lejos de las grandes ciudades, los pobladores de las comunidades están dedicados plenamente a la agricultura también se podría que antiguamente antes de ser comunidades era llamados huasipungos eran pequeñas parcelas de terreno donde las personas que vivían ahí realizando sembrío y en la crianza de animales domésticos pero ellos debían pagar por esas parcelas de terreno ellos para poder pagar trabajaban para los terratenientes de esa época pero mientras pasaban los años ellos lucharon para ser libres mediante años de lucha pudieron conformar como comunidades también en las comunidad de la sierra tienen varios elementos para poder conformar una comunidad entre ellas podemos recalcar que son: la posesión en común de algún recurso comunal, se encuentran conformadas por grupos de familias que actúan interactivamente para enfrentar situaciones y necesidades concretas, la relación de parentescos forman el tejido social y facilitan la fluidez de las relaciones cooperación e interacción estos son los elemento importantes para que se pueda conformar una comunidad donde los habitantes mediante ayudas entre ellos llamado mingas se ayudan y realizan trabajos agrícolas $\mathrm{u}$ otros $\mathrm{y}$ finalmente ellos tienen su propia forma de gobernar a los pobladores, la forma de la democracia, las leyes son muy diferentes donde ellos para poder dar una sanción por algún acto como el robo ellos realizan el llamado castigo indígena o limpia donde las personas que realizaron ese acto son sancionados y con la limpia para poder corregir que ya no incidan en el mismo acto las comunidades indígenas del Ecuador u otros país se rigen a las frases como: ama llulla ( no ser mentiroso), ama shuwa( no robar), ama killa(no ser ocioso) ellos tiene estas frases que usan si alguien de estas frases son infringidas pueden ser castigados en la Figura N.- 2 observaremos una de las comunidades de la parroquia zumbahua de donde varios habitantes han migrado hacia las grandes ciudades del Ecuador.

Figura N 2 Comunidad Michacalá

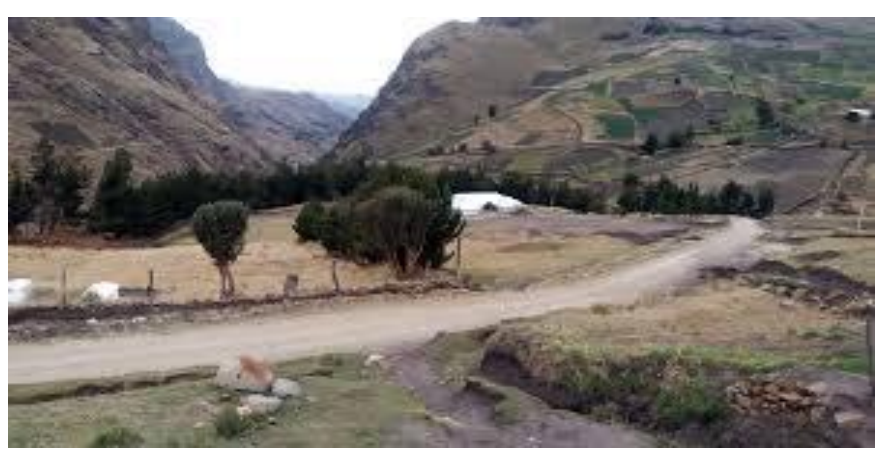

Fuente: Fotografía de la escuela Sergio Núñez 


\section{La migración}

Según los autores (Alejandro Canales y Christian Zlolniski 2014, Coronel 2011, Mario Vásquez \& Gustavo Coello) Se denomina migración del proceso mediante el que un individuo se desplaza de una zona geográfica hacia otra las personas que se desplazan son llamados migrantes, la migración es un proceso doloroso de abandono de su tierra natal por razones básicamente socio-económicas que impulsan a dejar sus familiares y amigos en busca de mejores condiciones de vida para él y su familia en este proceso migratorio implica dos conceptos: Emigración, que es la salida de personas de un país, lugar o región, para establecerse en otro país, lugar o región. La emigración implica una estimación negativa del nivel de vida de una persona y de su entorno familiar y una percepción de que al establecerse en otra parte aumentarán sus perspectivas económicas, sociales o de otro tipo o, por lo menos, de que sus esperanzas de una vida mejor se harán efectivas en el futuro.

Las migraciones constituyen un fenómeno demográfico sumamente complejo que responde a causas diversas y muy difíciles de determinar, en especial porque debido a las migraciones irregulares o disfrazadas de actividades turísticas o de otra índole, los datos cuantitativos son difíciles de obtener, especialmente en el caso de los países subdesarrollados las causas de la migración pueden ser: Causas políticas, Causas familiares, Causas culturales, Causas socioeconómicas estas mismas causas pueden tener sus consecuencias también podemos tener los diferentes efectos en la emigración nacional e internacional: Efectos demográficos, Efectos económicos, Efectos sociales también se podría decir que la Migración de miles de ecuatorianos se debe; al mal uso de los recursos que genera la economía ecuatoriana, a la incapacidad administrativa nacional, a la falta de compromiso de los políticos ecuatorianos y un alto índice de corrupción, lo que nos lleva a altas tasa de desempleo. Esto motiva a la decisión de migrar y mejorar su nivel y calidad de vida. Constantes son los intentos de salidas de los ecuatorianos hacia el extranjero, lo que es una respuesta a la permanencia de altas tasas de desempleo y subempleo en el país, productos de la insuficiente capacidad del aparato productivo ecuatoriano de absorber e insertar en su totalidad y de manera adecuada a la mano de obra disponible. El hecho es altamente preocupante porque a pesar de las migraciones de más de un millón de ecuatorianos desde 1990 hasta el 2010, las tasas de desempleo de subempleo no reducen.

En mi criterio diría que la migración es el desplazamiento de personas de un sitio de residencia o de sus comunidades en la mayor parte aquí en el Ecuador la migración ocurre en las comunidades rurales donde la calidad de vida no es buena el dinero que ellos adquieren mediante la agricultura no abastece para la subsistencia y la canasta básica de una familia es decir que mediante la agricultura ellos no obtienen ni un sueldo básico para poder sobrevivir dar estudio a sus hijo por esos motivos los padres salen a las grande ciudades donde ellos son explotados, discriminados o salen familias enteras donde la vida en las grandes ciudades muchas de ellos se han complicado ya por el crecimiento de la población los servicios básicos 
ya se han deteriorado poco a poco viven en lugares que no están acorde a sus necesidades en muchos de los casos los padres deberán abandonar su hogar para viajar al extranjero para poder trabajar y dar dinero para sus hijos para la comida, el estudio, la vestimenta así mismo para que ellos puedan tener una vida estable en la familia y las causas de la migración pueden ser causas políticas, familiares, culturales y socioeconómicas se podría decir que las causas políticas sería un ejemplo de nuestro país que la política de quienes dirigen nuestro países no es la correcta por la corrupción que hay por lo tanto la pobreza aumenta ya no existen muchos fuentes de trabajo ya sea en las ciudades peor en el campo por esos motivos algunos padres o miembro de la familia optan por salir del país para busca donde poder trabajar y dar una mejor vida a la familia, las causas familiares también es importante para poder tomar una decisión para poder emigrar a las ciudades extranjeras ya que en muchos de los cas se necesita la ayuda de familiares para poder establecer en un lugar y encontrar un sitio donde poder trabajar, las causas culturales para poder emigrar otros lugares muchas de las veces toca ver la cultura existente en el lugar como podría ser: el idioma, la religión, tradiciones y costumbres de una ciudad o país por las razones que muchas de las veces las familias migran para poder dar una mejor educación a los hijos y deberá ver esos aspectos para que tengan mayores posibilidades para educarse, las causas socioeconómicas la mayor parte de personas emigran por razones económicas buscando un mejor nivel de vida ya que en las comunidades el nivel de vida es una miseria en muchos de los casos eso se pasa en los países subdesarrollados donde el desempleo es en grandes cantidades y los migrantes deciden emigran al extranjero hasta arriesgando sus vidas solo por salir de su comunidad o su paisa para tener un nivel de vida mejor que las que ellos tienen muchos de estas migraciones también tienen sus consecuencias entre ellas sería el distanciamiento de familias por un largo periodo también hablaría en este caso de nuestro país el ecuador se muestra permanentemente conflictos político, crisis económica, entre otros no permite que las familias no tengan una mejor calidad de vida ni empleo y así poder reducir la tasa de desempleo y la migración pero en el 2007 se creó la Secretaria Nacional de Migrante para poder promover el regreso de ecuatorianos desde el extranjero donde dicha cartera de estado englobaron varias ayudas o así también la eliminación de muchos aranceles ya sea en el hogar, vehículos, dar empleos también hasta dar apoyo para que puedan adquirir su vivienda, en esta Figura N.-3 podemos observar la emigración o la entra de personas a nuestro país las ciudades que son como refugios así también los países de destino donde los migrantes optan viajar para tener un nivel de vida mejor. 
Figura N 3 Estructura del Sistema de Indicadores de Migración

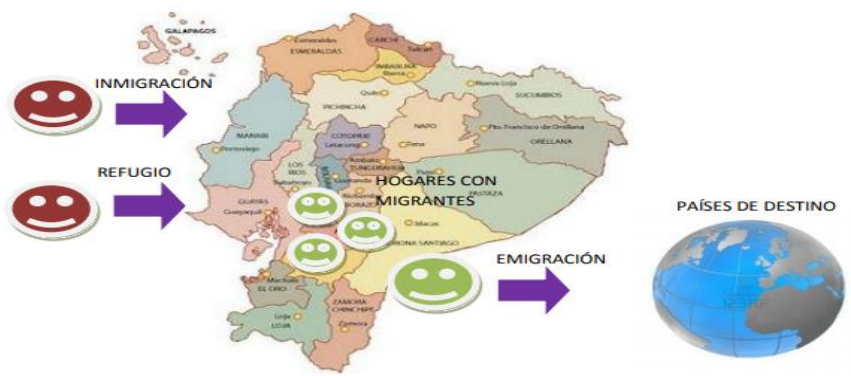

Fuente: Instituto Nacional de Estadísticas y Censo

\section{Las ciudades}

Según los autores (Romero 1976, Mumford 1970, Glaeser, 2012) Las ciudades son producto del tiempo, son los moldes en los cuales las vidas de los hombres se han enfriado y congelado dando lugar, por medio del arte, a formas duraderas, a momentos que de otro modo se desvanecerían al morir y no dejarían tras de sí posibilidades de renovación o de mayor participación. En la ciudad el tiempo se hace visible, los edificios, los monumentos y las vías públicas más accesibles que los registros escritos, y más a la vista de grandes cantidades de hombres que las construcciones dispersas del campo dejan una huella profunda incluso en la mente de los ignorantes o los indiferentes, el ser humano es social por naturaleza y donde mejor ejerce esa sociabilidad es en el núcleo urbano traza un nuevo camino que evidencia el triunfo de la ciudad en todo el mundo. Las ciudades surgen de las necesidades sociales del hombre y multiplican sus modos y sus métodos de expresión. En la ciudad, las fuerzas e influencias externas se mezclan con las locales: sus conflictos no son menos significativos que sus armonías. Y aquí, a través de la concentración de los medios de intercambio en el mercado y los lugares de encuentro, aparecen espontáneamente modos de vida alternativos: los caminos trillados de la aldea dejan de ser obligatorios y las metas ancestrales dejan de bastarse a sí mismas; hombres y mujeres desconocidos entre sí, intereses nuevos y dioses extranjeros, aflojan los tradicionales lazos de sangre y de vecindad. El desarrollo de los países depende casi por completo de sus ciudades y núcleos urbanos, es una realidad que la densidad urbana ofrece el camino más corto para pasar de la miseria a la prosperidad, de la inactividad al desarrollo, en el entorno urbano los choques fortuitos tienen consecuencias sociales, y las necesidades sociales pueden promover la aparición de inventos que conducirán a industrias y gobiernos a través de nuevas vías de experimentación. A veces la necesidad de un punto defensivo para protegerse del ataque de los depredadores hace que los habitantes de una aldea pasen a ocupar una colina fortificada, y del ineludible contacto que exige esta defensa surge la oportunidad de realizar intercambios más regulares y una cooperación más amplia. 
Según mi criterio las ciudades son grandes metrópolis a donde las personas emigran para poder tener una mejor calidad y para sus hijos dar una mejor educación se dice que las ciudades surgieron por las necesidades del hombre done ellos querían mejor el nivel de vida de ellos las grandes ciudades también ofrecen fuentes de trabajo mejores salarios en donde las personas que migran del campo pueden tener un mejor nivel de vida una buena educación el suficiente dinero para poder subsistir en su núcleo familiar también en las grandes metrópolis o ciudades una persona que haya emigrado desde un sector rural puede ser discriminado muchas de las personas han viajado a las ciudades con un solo fin empezar desde cero para cambiar su vida así también las grandes ciudades han sido como una competencia ya que día a día aparecerán nuevas ciudades que les obligaran a cambiar todo empezando desde los servicios básicos para dar una mejor imagen o que las personas residentes gocen de los mejores servicios en la ciudad y para que más personas llegue ya sea para residir o como visitantes en estas grandes ciudades muchas personas han empezado un sueño para tener un nivel de vida mejor cada día ira apareciendo nuevas ciudades que será creada por el hombre como una necesidad para sobrevivir y tener un nivel de vida estable, en las grandes ciudades los ruidos los choques serán algo del diario vivir ya que mientras crece la ciudad habrá nuevas cosas que el ser humado quienes viven se irían acostumbrando ya que la ciudad será un lugar donde todo ira sufriendo cambios evolucionara mientras el tiempo pasa así para poder ser una de las mejores ciudades y la metro poli más grande en este caso en nuestro país tenemos dos ciudades más grandes que son Guayaquil, Quito son las dos ciudades muy grandes donde los pobladores de todas las partes del Ecuador llegan para poder tener un cambio de vida Figura N.-4 aquí podemos observar una de las ciudades más grandes del Ecuador la gran cantidad de personas migrantes que se han concentrado.

Figura N 4 Quito concentración de migrantes de diferentes poblaciones

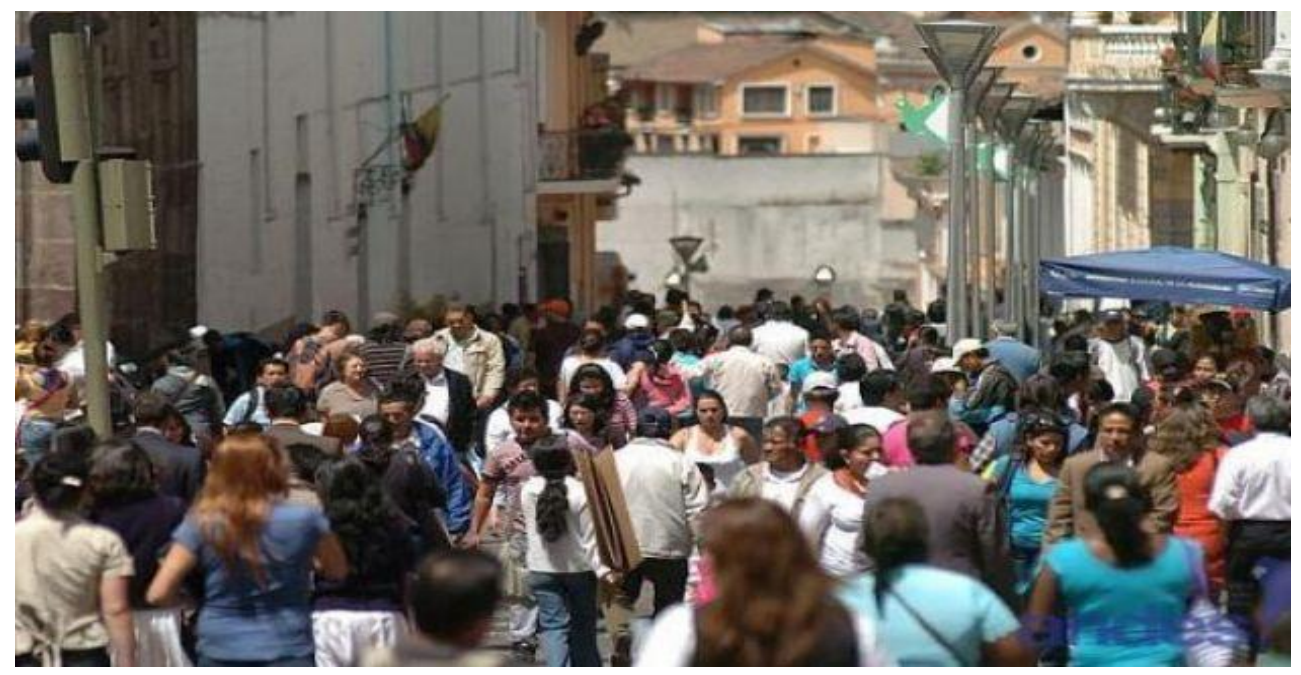

Fuente: El comercio 


\section{Conclusiones}

- El Mediantes esta investigación pude conocer cuántas personas salían de sus comunidades a las ciudades o al extranjero para poder tener mejores niveles de vida para mejor la economía de sus bolsillos y poder hacer estudiar o sus hijos o para la economía de su hogar.

- Recopile pequeños datos estadísticos del Instituto de Estadísticas y Censo para poder ver cuántas personas ha salido del país estos últimos años y así saber también que no existen muchas fuentes de empleo.

- Pude ver que muchas personas de las comunidades salen de su lugar de origen a las grandes ciudades para buscar nuevas fuentes de empleo para subsistir a la familia.

- Finalmente pude ver mediante esta investigación que la migración también puede traer consecuencias en la familia como la separación y por esta razón pueden las personas estar mal psicológica y emocionalmente.

\section{Referencias bibliográficas.}

Mario Vásquez \& Gustavo Coello. (s.f.). LA MIGRACION EN EL ECUADOR. En LA MIGRACION EN EL ECUADOR. MILAGRO.

Alejandro Canales y Christian Zlolniski. (2014). En COMUNIDADES TRANSNACIONALES Y MIGRACIÓN EN LA ERA DE LA GLOBALIZACIÓN. Mexico.

Altamirano, T. (2003). En T. Altamirano, El Perú y el Ecuador: Nuevos países de emigración.

Caussee Cathcart, M. (2009). Ciencias en su PC. (e. C. Sistema de Información Científica Red de Revistas Científicas de América Latina, Editor) Obtenido de http://www.redalyc.org/articulo.oa?id=181321553002

Cerrutti, M. (2005). En M. Cerrutti, La migración peruana a la Ciudad de Buenos Aires:su evolución y características. 
Vol. 1, N², p. 28-40, abril - junio, 2018

Coronel, J. (2011). En "ESTUDIO DEL IMPACTO DE LA MIGRACION EN LOS NUCLEOS FAMILIARES EN EL PAROQUIA SANTA ISABEL DURANTE EL PERIODO SEPTIEMBRE 2010 A JULIO DEL 2011”. Cuenca.

Facso Andes. (2008). En ECUADOR: La migración internacional en cifras.

Glaeser, E. (2012). En El triunfo de las ciudades. Obtenido de http://www.rniu.buap.mx/infoRNIU/feb12/2/lib_taurus_triunfo.pdf

Hidalgo, F. (2004). Migraciones. En Un juego con cartas marcadas . Quito.

Instituto Nacional de Estadística y Censos. (2018).

Marchioni, M. (1937). La intervención comunitaria en las nuevas condiciones sociales . Italia : Organización y desarrollo de la comunidad Marco Marchioni .

Mumford, L. (1970). En L. Mumford, La cultura de las ciudades.

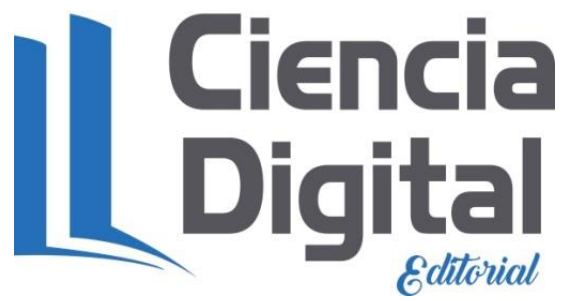


Para citar el artículo indexado.

Velasteguí López, E. (2018). La migración de personas de las comunidades a la ciudad. ConcienciaDigital, 1(2), 28-40. https://doi.org/10.33262/concienciadigital.v1i2.948

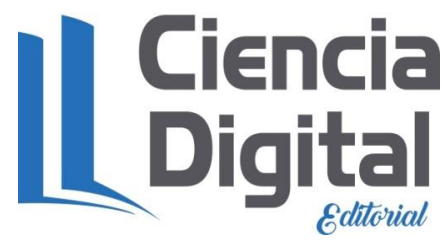

El artículo que se publica es de exclusiva responsabilidad de los autores y no necesariamente reflejan el pensamiento de la Revista Conciencia Digital.

El articulo queda en propiedad de la revista y, por tanto, su publicación parcial y/o total en otro medio tiene que ser autorizado por el director de la Revista Conciencia Digital.

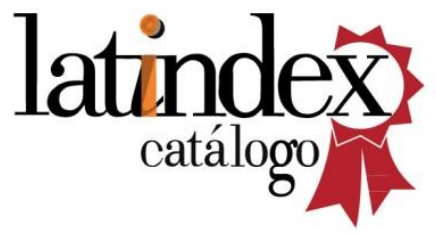

\section{Conciencia}

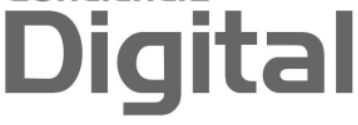

